\title{
High-throughput Proteomic Profiling of Male Breast Cancer Tissue
}

\author{
ELENI ZOGRAFOS ${ }^{1}$, STAVROS C. PROIKAKIS ${ }^{2}$, ATHANASIOS K. ANAGNOSTOPOULOS ${ }^{2}$, \\ ANNA-MARIA KORAKITI ${ }^{3}$, FLORA ZAGOURI $^{3}$, MARIA GAZOULI ${ }^{1}$ and GEORGE T. TSANGARIS ${ }^{2}$ \\ ${ }^{1}$ Department of Basic Medical Sciences, Laboratory of Biology, School of Medicine, \\ National and Kapodistrian University of Athens, Athens, Greece; \\ ${ }^{2}$ Proteomics Research Unit, Center of Basic Research II, \\ Biomedical Research Foundation of the Academy of Athens, Athens, Greece; \\ ${ }^{3}$ Department of Clinical Therapeutics, Alexandra Hospital, School of Medicine, \\ National and Kapodistrian University of Athens, Athens, Greece
}

\begin{abstract}
Background/Aim: Until now, little emphasis has been placed on the protein expression profile of male breast cancer $(M B C)$ tumors, due to the rarity of the disease. The present study aimed to identify a proteomic pattern that is characteristic for malignant male breast tissue epithelium. Materials and Methods: The protein content of four male breast tumors and corresponding adjacent healthy (control) tissues was analyzed by high-throughput nano-liquid chromatography-MS/MS technology. Results: A total of 2,352 proteins were identified, that correspond to 1,249 single gene products, with diverse biological roles. Of those, a panel of 119 differentially expressed tissue proteins was identified in $M B C$ samples compared to controls; 90 were found to be over-expressed in MBC tissues, while 29 were downregulated. Concurrently, 844 proteins were detected only in $M B C$ tumors and 197 were expressed exclusively in control mammary samples. Conclusion: Differential proteomic expression was found in MBC tissue, leading to improved understanding of $M B C$ pathology and highlighting the need for personalized management of male patients.
\end{abstract}

Male breast cancer (MBC) is an uncommon disease, accounting for fewer than $1 \%$ of all diagnosed breast

This article is freely accessible online.

Correspondence to: Eleni Zografos, BSc, MD, MSc, Ph.D., Department of Basic Medical Sciences, School of Medicine, University of Athens, Michalakopoulou 176, 11527 Athens, Greece. Tel: +30 2107462231,+30 2107462244, e-mail: el_zogra@hotmail.com

Key Words: Male breast cancer, proteomics, high resolution mass spectrometry, HPLC-MS/MS. carcinoma cases (1). The estimates of the American Cancer Society, that predict merely 2,650 new cases of invasive MBC and 530 deaths from the disease in the United States for 2021, indicate its limited impact on male morbidity and mortality (2). Despite the fact that this entity is rare, the incidence of $\mathrm{MBC}$ is on the rise over the past two decades (3), attracting increasing interest in terms of deciphering its biology, etiopathogenesis, clinical presentation and unique management. Still, because of the low number of diagnoses, MBC remains understudied, prospective research-based progress is hindered, and decision-making for patients and physicians is based on observational retrospective studies $(4,5)$.

Owning to the scarcity of research in the field, clinical data on the management of MBC are usually extrapolated from trials on its female counterpart; this approach is becoming less and less acceptable due to the inherent discrepancies between the two entities. Characteristically, the largest retrospective collection and pathological review of MBC tumor samples so far, showed a lack of association between histological grading and outcome, which may be linked to the different distribution of disease subtypes in men compared with women (6). For example, men are much more likely to express the estrogen (ER) or androgen (AR) receptors than women, and less likely to over-express HER2 $(6,7)$. Lobular tumors are also much less frequent in men despite being relatively common in women, whereas most MBC cases are subtyped as either Luminal A-like or Luminal-B-like/HER-2-negative $(6,8)$. The genomic landscape of the disease further highlights the differences between sexes, with a recent publication reporting less frequent 16q losses, PIK3CA mutations, and TP53 mutations than those seen in ER-positive/HER2-negative female breast cancers, but more frequent somatic mutations in genes involved in DNA repair pathways (9). These findings do not 
only highlight the biologic heterogeneity between male and female patients, but also indicate the need for a better tailored approach for optimal male breast cancer classification and prognostication.

Current efforts are focusing on the identification of unique subgroups of breast malignancies in men, which differ from the intrinsic subtypes women harbor, mainly in the context of gene expression studies (10). Characteristically, gene expression profiling approaches have geared towards the stratification of MBC into two distinct subgroups that occur only among males and that differ from the well-established intrinsic subtypes of breast cancer in women; one group displays high expression of immune response genes and genes associated with ER signaling, whereas the other subgroup shows up-regulation of genes involved in activation of invasion/metastasis and induction of angiogenesis, such as $H O X B 7$ (11). However, genes lack any catalytic functions and instead exercise their effects through proteins, the biomolecules that direct cellular processes and disease progression (12). Given the weak link between genomic alterations and protein functionality, due to biological processes such as RNA alternative splicing and post-translational modifications, using targeted proteomics in clinical settings allows us to gain a deeper molecular knowledge of breast cancer and shed light on biomarker research (13). Recent advances in proteomic technologies, such as High Performance Liquid Chromatography-tandem Mass Spectrometry (HPLC-MS/MS), enable us to combine high separation capacity and strong qualitative ability of proteins in biological samples (14).

In the current study, we employed HPLC-MS/MS to examine protein alterations in MBC tissue samples. HPLCMS/MS is proposed as a powerful analytical tool for deciphering protein expression alterations as a function of breast cancer progression and, according to our knowledge, has not yet been utilized for the proteomic profiling of male breast cancer tumors. Our aim was to identify a panel of proteins that are differentially regulated in male breast tumors, by comparing protein profiles between tumor and adjacent non-tumor tissues. Unravelling the altered protein expression in malignant tissue could help researchers better understand the pathogenesis of male breast cancer and contribute towards the identification of more accurate markers that have the potential to be clinically meaningful.

\section{Materials and Methods}

Patients and tissue samples collection. Four male patients with sporadic breast cancer (MBC) attending the First Propaedeutic Surgical Department at Hippokration General Hospital in Athens, Greece, were enrolled in this study. Participation was voluntary and informed consent was provided by each participant. MBC was diagnosed based on a combination of standard clinical, radiological, and histological criteria (15). Breast tissue samples were collected
Table I. Clinical characteristics of the four male breast cancer patients.

\begin{tabular}{lcccc}
\hline Variable & \multicolumn{4}{c}{ Patient number } \\
\cline { 2 - 5 } & $\# 1$ & $\# 2$ & $\# 3$ & $\# 4$ \\
\hline Age at Diagnosis & 87 & 82 & 70 & 65 \\
Ethnicity & Greek & Greek & Greek & Albanian \\
Side & Right & Left & Right & Right \\
Tumor size & $1.5 \mathrm{~cm}$ & $2.1 \mathrm{~cm}$ & $1.2 \mathrm{~cm}$ & $1.7 \mathrm{~cm}$ \\
Tumor type & Ductal & Ductal & Ductal & Ductal \\
Grade & 2 & 3 & 2 & 2 \\
ER & $80 \%$ & $70 \%$ & $99 \%$ & - \\
PR & $80 \%$ & $80 \%$ & $95 \%$ & - \\
HER2/neu & - & $2+$ & - & $1+$ \\
Ki-67 & $20-25 \%$ & $30 \%$ & $25 \%$ & $25 \%$ \\
Lymph nodes & $\mathrm{N} 0$ & $\mathrm{~N} 1$ & $\mathrm{~N} 0$ & $\mathrm{~N} 0$ \\
\hline
\end{tabular}

from each patient at the time of surgery and stored at $-80^{\circ} \mathrm{C}$ until use. The control group consisted of an equal number of adjacent healthy tissue samples, which were extracted from each patient during surgical treatment and preserved at the same storage conditions as the malignant tumors. The study was approved by the Ethical Committee of the participating hospital. The main demographic and clinical characteristics of the MBC patients are presented in Table I.

Tissue sample preparation and peptide generation. The fresh frozen tissue samples were treated with lysis buffer, as previously described (16). Following lysis, tissue disruption was carried out, first by mechanical pressure and then by tip-sonication for three cycles of $18 \mathrm{~s}$ duration each, under an amplification of $38 \%$. The subsequent step involved centrifugation for $20 \mathrm{~min}$ at $13,000 \times \mathrm{g}$, in order to discard the pellet which contained organelle debris and other insoluble materials. The Bradford protein assay was applied for total protein quantification of the supernatant. Then, $200 \mu \mathrm{g}$ of protein from each tissue sample was treated with $7 \mathrm{M}$ urea buffer and $80 \mathrm{mM}$ triethylamonium bicarbonate. Next, protein alkylation was performed applying $55 \mathrm{mM}$ iodoacetamide solution for $30 \mathrm{~min}$ at room temperature in dark conditions. Finally, a trypsin solution (Roche Diagnostics, Hoffman-La-Roche, Basel, Switzerland) at a final concentration of $500 \mathrm{ng} / \mu \mathrm{l}$ was added to all samples, and after thorough mixing, the digestion took place overnight, at $36 \square \mathrm{C}$, in a humidified environment. The following day, 5\% (vol/vol) acetic acid was added to end trypsinization, and the peptide-containing mixtures were lyophilized in a vacuum concentrator for approximately $1 \mathrm{~h}$, until they were completely dried. The resulting powder was dissolved in $0.1 \%$ formic acid in distilled water and LC-MS/MS analysis followed.

One-dimensional nano liquid chromatography (1D-nanoLC) separation and MS/MS analysis. MS analysis was performed as previously described (17). The peptides that derived from the previous steps were analyzed using an LTQ Orbitrap Elite coupled with a Dionex Ultimate-3000 nanoHPLC system (Thermo Scientific, Rockford, IL, USA). Specifically, one-dimensional liquid chromatography (1D-LC) separation took place after loading our samples subsequently onto two C-18 pre-columns (Thermo 
Scientific, PepMap ${ }^{\circledR}$ RSLC, $100 \AA$ A, 3- $\mu$ m-bead-packed 15-cm column and $2-\mu \mathrm{m}$-bead-packed $50-\mathrm{cm}$ column). The mobile phases $\mathrm{A}$ and $\mathrm{B}$ were $0.1 \%$ formic acid and $99 \%$ acetonitrile in water, respectively, at a flow rate of $0.3 \mu \mathrm{l} / \mathrm{min}$. The gradient elution profile for all runs was 360 min for $2 \%-30 \%$ of buffer B $(99.9 \%$ acetonitrile, $0.1 \%$ formic acid) at a constant $300 \mathrm{nl} / \mathrm{min}$ flow rate. Notably, to achieve maximum peptide recovery, we ended up testing several linear and non-linear gradient profiles, aiming at the optimal gradient, according to the material under study.

In the following step, an Orbitrap Elite mass spectrometer fitted with a nano-spray source was employed to collect mass spectra; data were acquired in accordance to a data-dependent acquisition strategy. Full-scan data were collected with a resolution of 60,000 and a maximum injection time of $120 \mathrm{~ms}$. Tandem mass spectrometry for the 20 most intense ions per survey scan was performed, and peptide fragmentation was achieved at a high-energy collision dissociation fragmentation mode, set at a normalized collision energy of $36 \mathrm{NSE} \%$. The MS/MS spectra of the resulting fragments were acquired with a resolving power of 15,000 and a maximum integration time of $120 \mathrm{~ms}$. Measurements were performed using m/z 445.120025 as lock mass. Notably, to prevent repetitive selection of the same peptide, dynamic exclusion was employed within $45 \mathrm{~s}$.

Data analysis. The raw data files that arose were processed with the Proteome Discoverer software (Thermo Scientific), using the SequestHT (version 28.0, Thermo Scientific) search engine applying the Homo sapiens UniProtKB/Swiss-Prot database. MS/MS searches were performed according to specific parameters; these included a $10 \mathrm{ppm}$ parent peptide mass tolerance, a 0.05 fragment ion tolerance, up to two missed cleavage points for trypsin, cysteine methylation as a fixed modification, and oxidation of methionine as a variable modification. Peptide spectral matches (PSM) were considered valid at a $1 \%$ false discovery rate (FDR) (q-value $<0.01)$. Further peptide filtering was done based on Xcorr versus peptide charge values (percolator maximum Delta $\mathrm{Cn}$ was 0.05). Of note, the minimum length of acceptable identified peptides was set as six amino acids.

Protein annotation and network analysis. Information on the identified proteins' annotation were retrieved via the Proteome Discoverer software. Gene Ontology (GO) terms regarding biological process and pathway was used for the construction of protein classifications, while they were retrieved through PANTHER (Protein Analysis Through Evolutionary Relationships) version 16, which is an integrated knowledgebase of evolutionary and functional relationships between protein-coding genes (18). The potential interactions across previously detected proteins were studied with the STRING webserver.

\section{Results}

Focusing on deciphering the proteome of male breast cancer tumors at a high-resolution scale, four male breast cancer tumor and four adjacent normal tissue samples from the same patients were analyzed using untargeted tandem mass spectrometry. A total of 2,352 proteins were identified in the analyzed tissue samples, that correspond to 1,249 single gene products with diverse biological roles (Supplementary Tables I-IV). Of those, a panel of 119 differentially expressed tissue proteins was identified in MBC samples compared to controls; 90 were found to be over-expressed in MBC tissues, while 29 were down-regulated in male breast tumors. Concurrently, 844 proteins were detected exclusively in the MBC group and 197 were only expressed in the control samples.

The pathways which engage the differentially expressed proteins identified in our samples were studied using the STRING database (Figure 1, Figure 2, Figure 3 and Figure 4). These figures depict a non-directed graph with the functional interactions in the dataset presented as predicted by STRING. Each node represents a protein, and an edge between protein $\mathrm{X}$ and $\mathrm{Y}$ represents predicted functional interaction among these proteins. This interplay can be viewed as one protein being regulated by another, a genetic or physical interaction, the participation in the same biological process, or the common regulation of both proteins by a third molecule, among others. Thicker network lines demonstrate stronger protein relation as well as neighboring positions.

For this interpretation, a multi-level comprehensive analysis based on GO categorization was performed using the PANTHER classification system, which sorts the proteins into respective groups based on their biological process, and their involvement in different signaling pathways. Concerning the 90 over-expressed proteins in MBC tumors, as presented in Figure 5, it is of great interest that the four major groups involved molecules related to cellular process $(52.9 \%)$, metabolic process proteins $(28.7 \%)$, biological regulation $(17.2 \%)$, and members of the response to stimulus process $(14.9 \%)$, factors that are known to be aberrant in cancer. Regarding the analysis of the 29 down-regulated proteins, as illustrated in Figure 5, the majority of them are involved in cellular process $(37.9 \%)$, biological regulation $(31.0 \%)$, metabolic process $(31.0 \%)$, and response to stimulus (27.6\%). Additionally, as shown in Figure 6, most of the 844 proteins identified as being exclusively expressed in $\mathrm{MBC}$ tumors participate in cellular processes $(55.5 \%)$, metabolic processes $(34.7 \%)$, and biological regulation $(22.2 \%)$. Finally, the correlation of the 197 proteins identified exclusively in the control samples to biological processes, according to the GO categorization, revealed that half of the proteins identified were involved in cellular process $(53.6 \%)$, followed by a significant number of proteins taking part in the metabolic process $(29.3 \%)$ and the biological regulation $(28.7 \%$ ) (Figure 6).

Regarding the PANTHER pathway analysis, as illustrated in Figure 7 and Figure 8, several predominant pathways were identified in each group of proteins. Specifically, $10.3 \%$ of the over-expressed and $17.2 \%$ of the down-regulated proteins in $\mathrm{MBC}$ corresponded to proteins found in the integrin signaling pathway, demonstrating the importance of the crosstalk between cancer cells and their microenvironment that triggers a variety of critical signaling cues (Figure 7). 


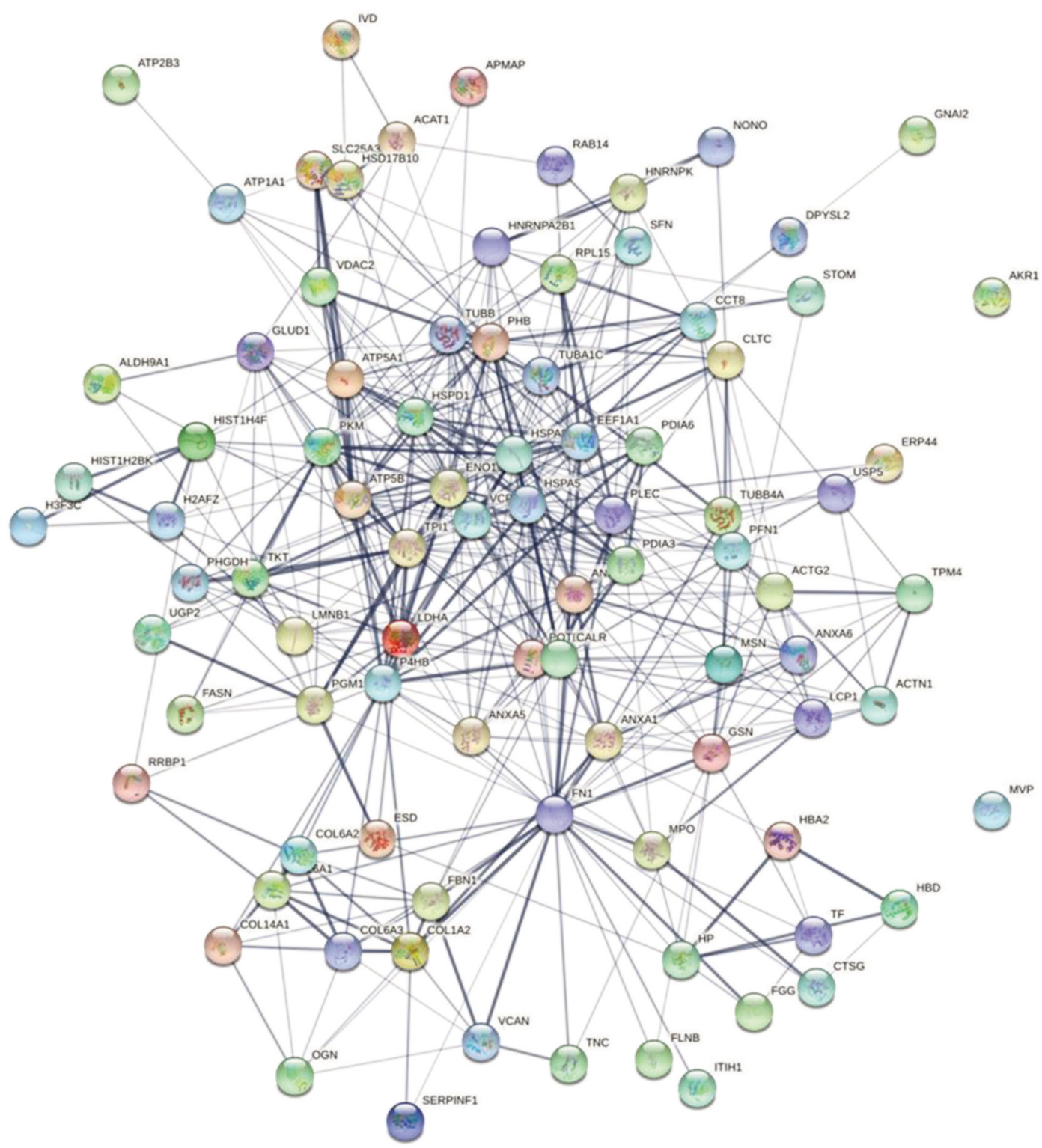

Figure 1. The figure depicts the interactions of the 90 proteins identified as being over-expressed in male breast cancer (MBC) samples. Each node represents a protein, Edges represent functional interaction between the proteins. Thicker network lines demonstrate stronger protein relation as well as neighboring positions. Protein abbreviations used are presented in Supplementary Table I.

With regards to the proteins found exclusively in MBC samples, $2.6 \%$ were involved in the Huntington disease pathway, $2.2 \%$ in the Wnt signaling pathway, $2.2 \%$ in the inflammation mediated by chemokine and cytokine signaling pathway, and $1.9 \%$ in the integrin signaling pathway (Figure 8). Finally, a significant number of proteins found exclusively in the control samples were implicated in the integrin signaling pathway (3.3\%), in cytoskeletal regulation by Rho GTPase $(2.8 \%)$, in inflammation mediated by chemokine and cytokine signaling pathway $(2.8 \%)$, as well as in the heterotrimeric G-protein signaling pathway-Gi alpha and Gs alpha mediated pathway (2.8\%) (Figure 8).

Based on the interactions of differentially expressed proteins in cancerous and non-cancerous tissues, distinct protein groups with different biological processes are observed. The potential interactions across upregulated proteins in $\mathrm{MBC}$, revealed several proteins in the center of the network that demonstrated stronger relations (Figure 1). These proteins are mainly involved in glycolysis (ENO1, TPI1, PKM), heat stress response (ANXA2) and chaperonin- 


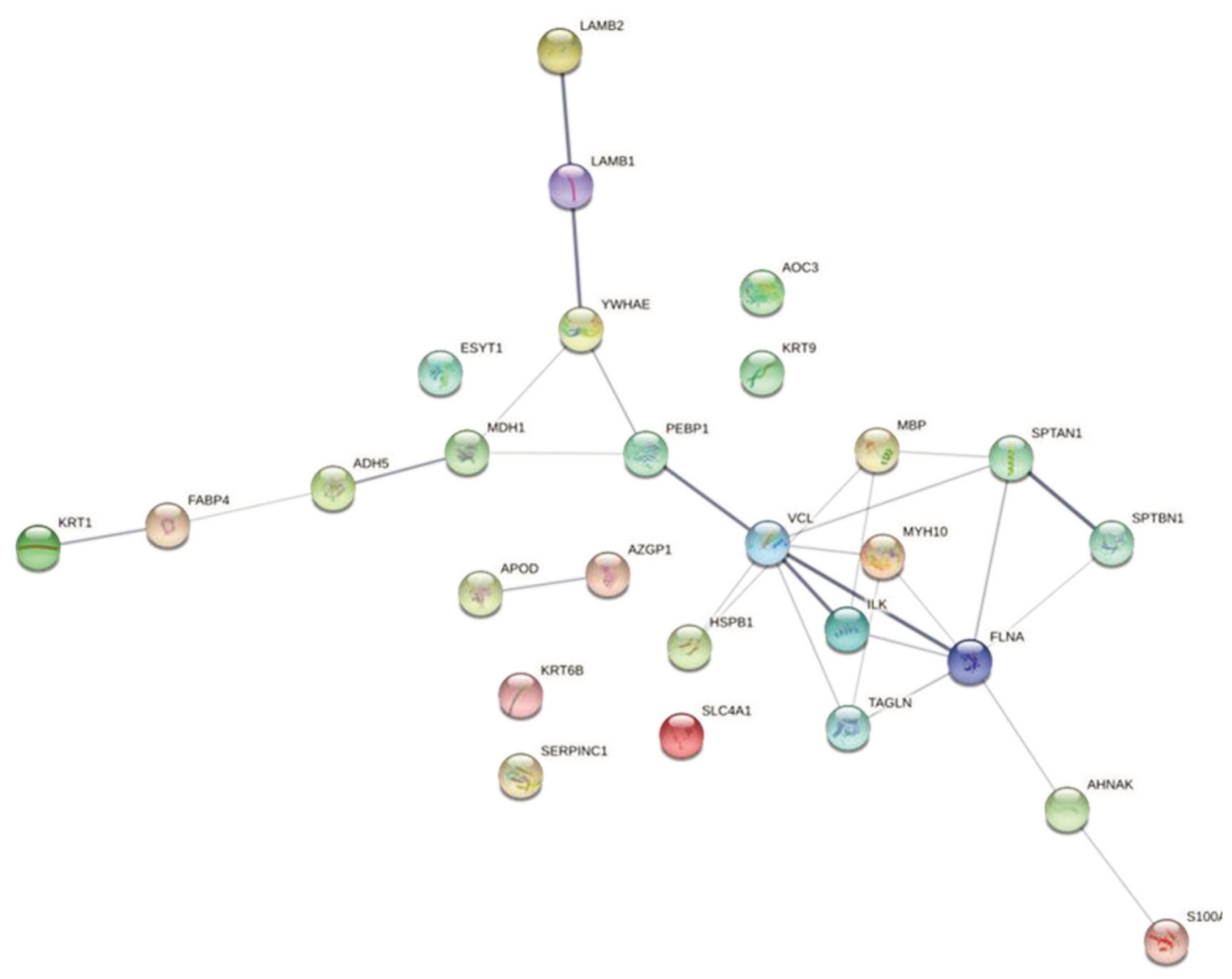

Figure 2. STRING network of the 29 proteins that were down-regulated in male breast tumors. Network nodes represent proteins and edges represent protein-protein associations.

mediated protein folding (HSPA5, HSPA8, HSPD1, VCP, PDIA3, PDIA6), protein biosynthesis (EEF1A1), cytoskeleton organization (PLEC), DNA synthesis (PHB), cell division (TUBB, TUBA1C) and ATP synthesis (ATP5F1A, ATP5F1B). Concerning the interactions among proteins down-regulated in $\mathrm{MBC}$ samples, the densest area presented in Figure 2, involved molecules associated with the integrin- and growth factor-signaling pathway (ILK), cell adhesion (VCL, MYH10), myelination (MBP), cytoskeleton organization (SPTAN1, SPTBN1, FLNA), and epithelial cell differentiation (TAGLN). Additionally, the network of the interactions among the proteins exclusively identified in MBC (Figure 3) revealed distinct subgroups involved in protein biosynthesis and transport, proteasome assembly, mRNA processing, cell adhesion, endocytosis, cell division, and mitochondrial electron transport chain. Finally, the functional relationships analysis of the proteins expressed only in control samples, resulted in a network with distinct areas encompassing proteins involved in cell adhesion (LAMC1, LAMA4, LAMA5, WVF, NID1, ICAM1, MCAM,
CD99), in glycolysis/glycogen metabolism/glyconeogenesis (PYGL, PYGB, ALDOC, HK2, BPGM, GPD1), and in translation/protein biosynthesis/proteasome assembly (RPL19, RPL29, EIF3H, ADRM1, PSMA6) (Figure 4).

\section{Discussion}

In the current study, we attempted to characterize the tissue proteomic profile of patients with MBC using highresolution mass spectrometric technology. The analysis identified a total of 2,352 proteins, belonging to 1,249 single gene products, that are involved in a multitude of biological processes. Several proteins (119 molecules) were differentially expressed in patients with MBC compared to controls. Of those, 90 were found to be upregulated in all tissue samples of the MBC group, while 29 were downregulated in male breast tumors. Additionally, 844 proteins were detected only in samples from MBC tumors and 197 were exclusively expressed in the control samples. The comparison revealed outstanding differences between MBC 


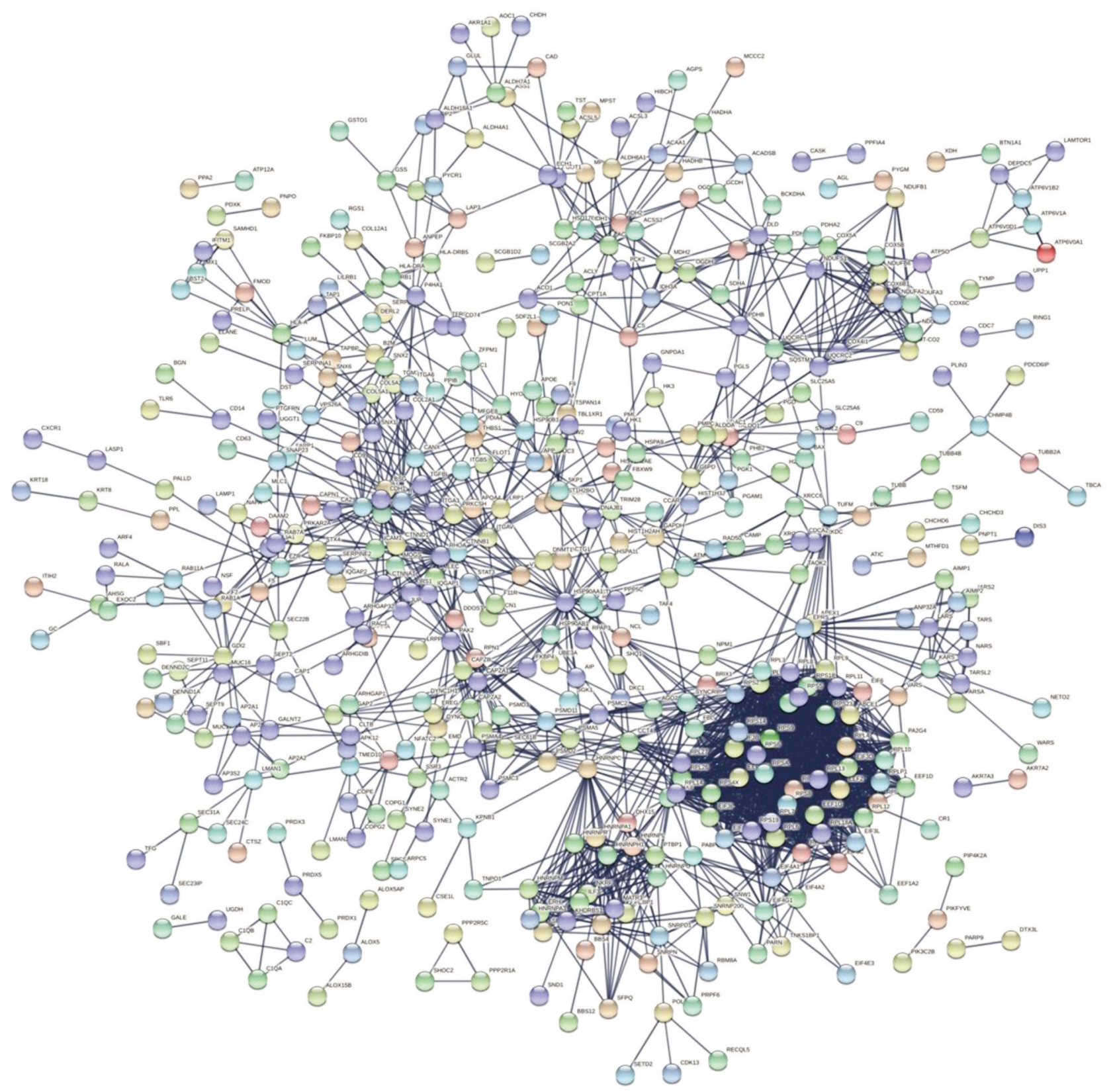

Figure 3. A total of 844 proteins were detected only in the male breast cancer $(M B C)$ group. Due to the large number of identified molecules, the STRING network of these proteins was set at a minimum required interaction score of highest confidence (0.900), with no more than five interactors for each protein shown, while the disconnected nodes in the network were hidden.

samples and adjacent "healthy" tissue from the same patient, giving us insight into the perturbations of pathways within tumor cells and establishing an important foundation for future male breast cancer-related discoveries using differential proteomics profiling.

MS technologies have enabled increased proteome coverage rather than using the traditional approach of translating gene expression data. Especially in the context of clinical proteomics, cancerous tissue proteomic analysis provides the most accurate reflection of the tumor's physiological state (1922). However, most protein MS-based breast cancer screening strategies refer to female patients and although possible biomarkers have been identified, few have been validated so as to achieve clinical application (23-26). Regarding protein- 


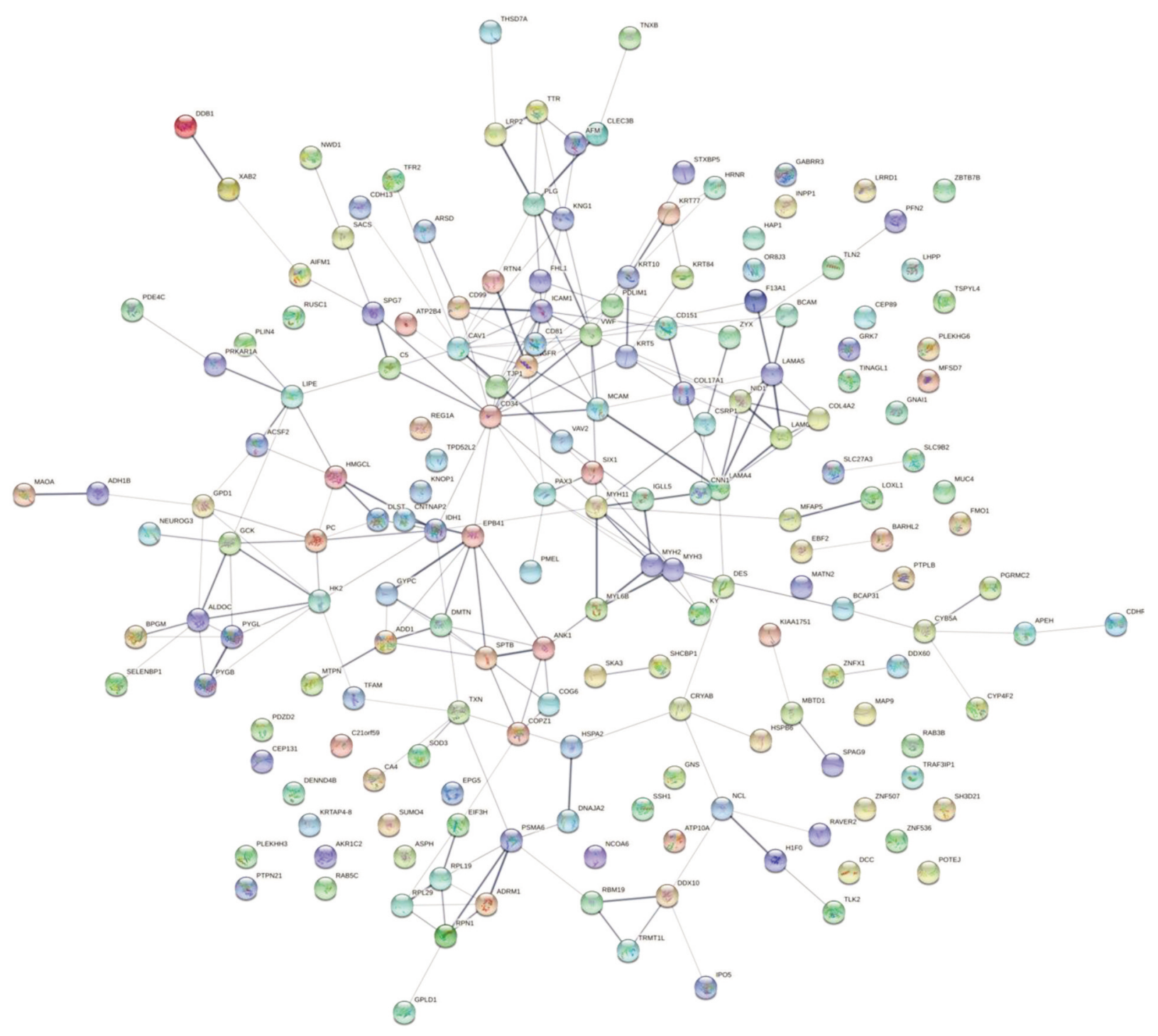

Figure 4. The figure depicts the interactions of the 197 proteins identified as being exclusively expressed in our control samples. Network nodes represent proteins. Thicker network lines demonstrate stronger protein relation as well as neighboring positions.

expression alterations in male breast tumors, the only published study using MALDI-TOF mass spectrometric technology revealed over-expression of structural proteins, glycolytic enzymes, stress related proteins, enzymes involved in the synthesis of AMP, heat-shock proteins, and others (27). However, further advancements in the HPLC-MS/MS platform, which exhibits increased sensitivity and reliability in identifying proteins in complex mixtures, have not yet been utilized for the proteomic profiling of cancerous male breast regions that are obtained during surgery.

Breast cancer has traditionally been considered a femalespecific disease, presenting approximately one hundred times more often in women compared to men (28). To date, no proteomic approach has identified the molecular mechanisms involved in gender-specific tumorigenesis - much is still to be learned. The hypothesis is that by looking at gender-related proteins, physiological differences between men and women could be revealed. Characteristically, none of the differentially expressed proteins that we identified are encoded by genes on the $\mathrm{Y}$ chromosome. On the other hand, a small number of over-expressed proteins in $\mathrm{MBC}$ are products of genes on the single $\mathrm{X}$ chromosome in males (Table II). Of these, the cytoskeletal protein MOES has been suggested as a potential marker for poor prognosis in females with ER-positive breast 


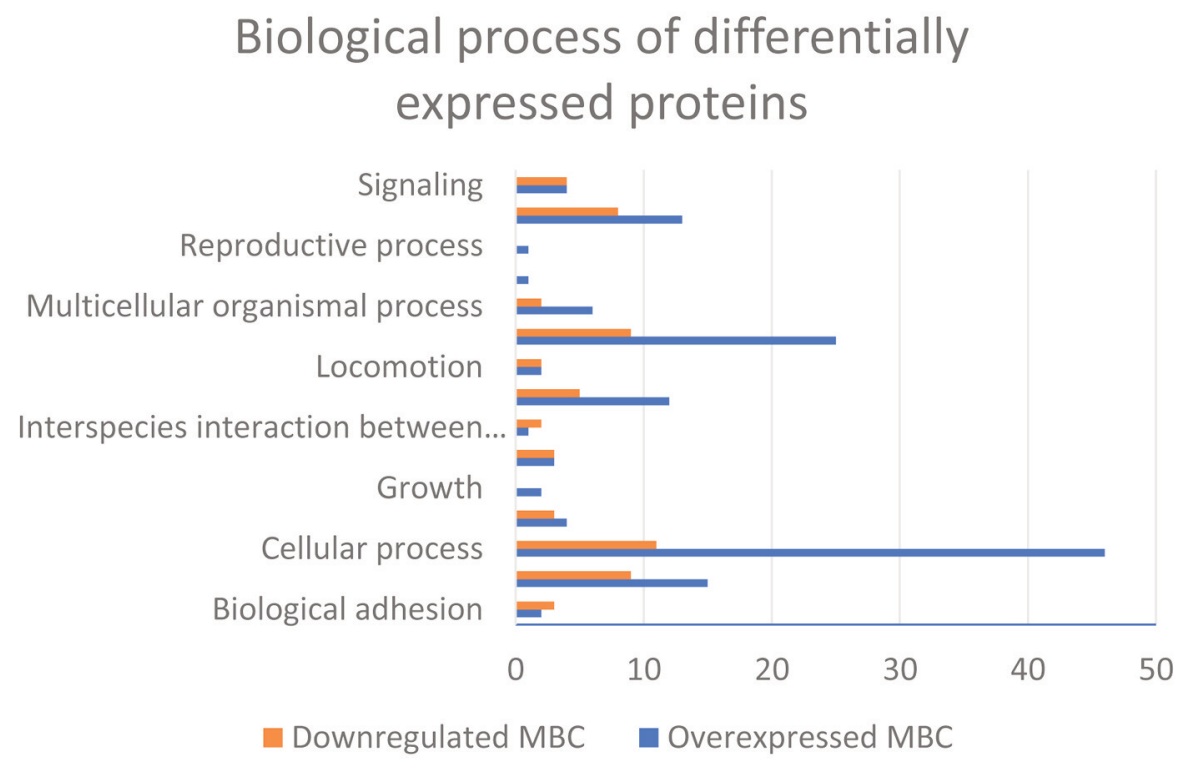

Figure 5. Biological process of differentially expressed proteins, either over-expressed in male breast cancer (MBC) tissues (blue color) or downregulated in MBC tissues (orange color).

\section{Biological process of proteins exclusively in MBC or CONTROL}

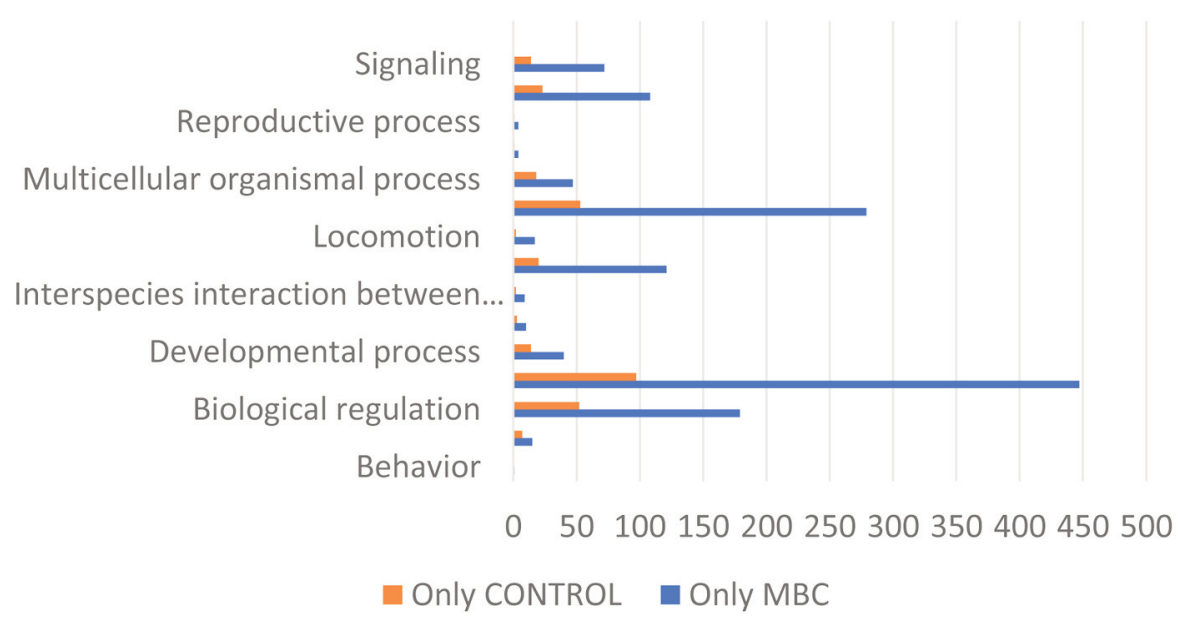

Figure 6. Biological process of proteins exclusively expressed in male breast cancer (MBC) (blue color) or CONTROL (orange color).

cancer treated with tamoxifen (29). HCD2 is a lipid metabolism- mitochondrial-related protein that has been found to be selectively upregulated in mammosphere-forming cells from breast tumor cell lines that represent the population of cancer stem cells (CSCs) that might lead to the failure of routine chemotherapy and the recurrence of the disease (30). NONO is a DNA-binding protein found in a number of breast tumor cell lines, and has been proposed as a significant independent prognostic factor for patients since its knockdown substantially represses the proliferation of breast cancer cells, acting through the pre-mRNA splicing of cell proliferationrelated genes (31). Interestingly, NONO exhibits gender expression differences since higher expression levels in females have been reported (32). On the other hand, FLNA is 


\section{Pathway analysis of differentially expressed proteins in MBC}

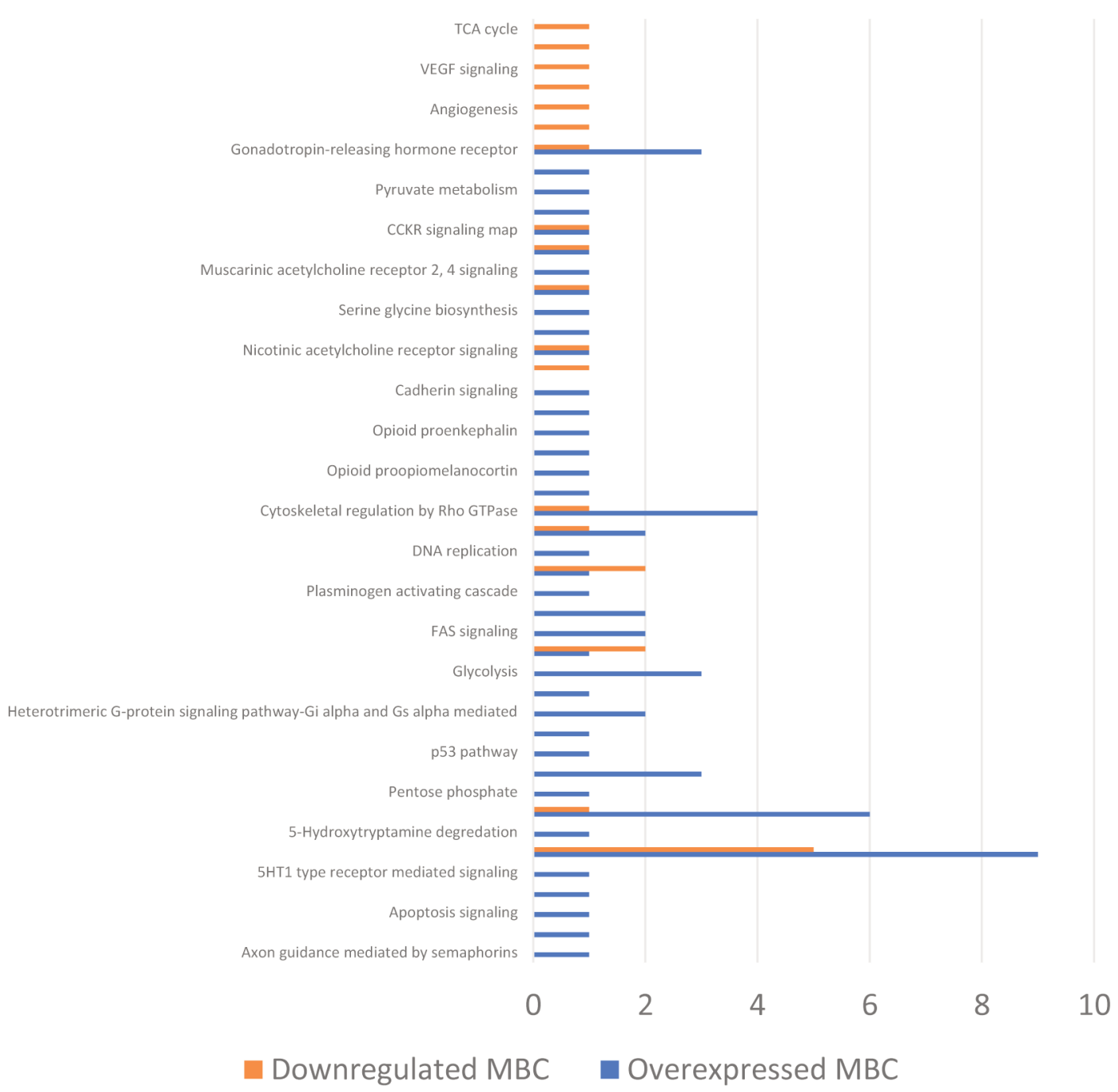

Figure 7. Pathway analysis of differentially expressed proteins in male breast cancer (MBC).

an X-linked protein that we found to be down-regulated in MBC tissues. Although most of the studies point to a positive correlation between expression of FLNA and breast cancer development and progression (33), some studies are in line with our results, showing that FLNA expression is negatively correlated with the metastatic potential of breast cancer lines $(34,35)$. This is probably because FLNA is able to regulate focal adhesion disassembly and suppress breast cancer cell migration and invasion (35). These gender differences could have important clinical implications for treatment decision making and might eventually enable us to make better treatment choices, individualized for each patient.
Concerning the limitations of our study that deserve mention, it is important to advert that we analyzed a small number of tumor samples due to the rarity of the disease, deeming our results merely indicative and in need of further confirmation in a larger cohort of patients. Despite the small number of samples analyzed and thus our inability to draw safe conclusions on the detailed proteomic pattern of $\mathrm{MBC}$ tumors, we obtained high-throughput reproducible results that literature is currently lacking.

In conclusion, in this work we detected the differentially expressed protein in MBC tumors, using high resolution MS. The data generated highlight the important contribution of 


\section{Pathway analysis of proteins exclusively in MBC or CONTROL}

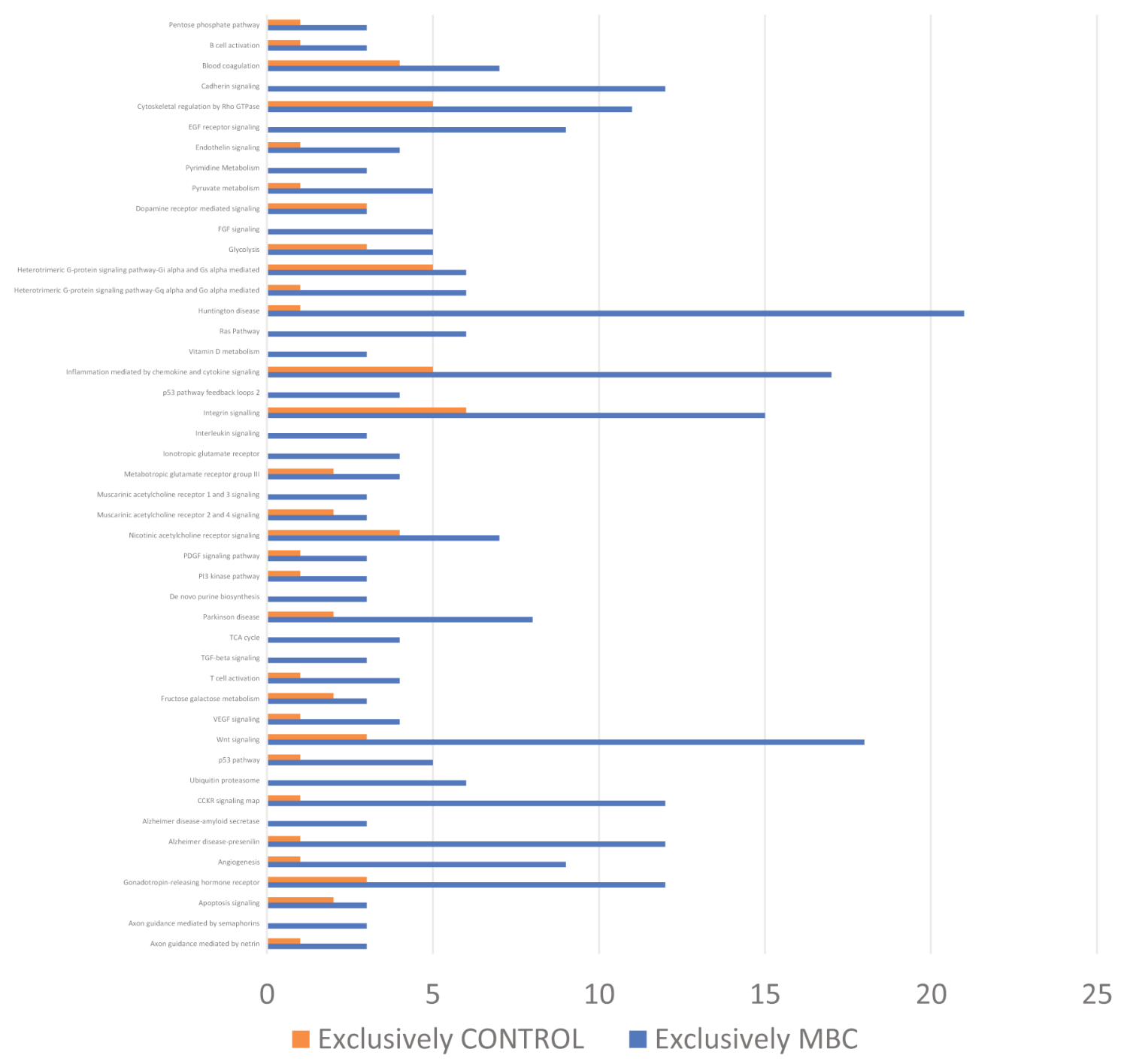

Figure 8. Pathway analysis of proteins exclusively expressed in male breast cancer (MBC) tissues (blue color) or CONTROL tissue samples (orange color). This figure depicts the signal transduction pathways in which more than three of the identified proteins are involved in.

Table II. Differentially expressed proteins in male breast cancer (MBC) encoded by the X chromosome genes.

\begin{tabular}{ll}
\hline & X Chromosome proteins \\
\hline Over-expressed MBC & MOES_HUMAN, NONO_HUMAN, HCD2_HUMAN \\
Down-regulated MBC & FLNA_HUMAN \\
Expressed only in MBC & CSKP_HUMAN, NKRF_HUMAN, MBTP2_HUMAN, RPX2_HUMAN, DKC1_HUMAN, PGK1_HUMAN, \\
& FA9_HUMAN, ADT2_HUMAN, ODPA_HUMAN, G6PD_HUMAN, ADT3_HUMAN, PGS1_HUMAN, \\
& RL10_HUMAN, SMCA1_HUMAN, V2R_HUMAN, ABCD1_HUMAN, EMD_HUMAN, FGD1_HUMAN, \\
& DHD1_HUMAN, PRDX4_HUMAN, T2B3_HUMAN, SATL1_HUMAN, CTT1_HUMAN, CAL4_HUMAN, \\
& OTUD5_HUMAN, LH15_HUMAN, APOO_HUMAN \\
Expressed only in control & A6NCN8_HUMAN, AIFM1_HUMAN, CD99_HUMAN, AOFA_HUMAN, BAP31_HUMAN, \\
& FHL1_HUMAN, S27A3_HUMAN \\
\hline
\end{tabular}


MS-based techniques towards deciphering the molecular basis of male breast malignancies. While some strides have been made to address the need for male-specific information, the challenges still exist and need to be tackled from diverse perspectives and on a larger scale. The in-depth proteomic profiling of this rare population might ensure that MBC patients receive the most appropriate treatment in respect to the gender specific differences in cancer biology and management.

\section{Supplementary Material}

The Supplementary Tables I to IV can be accessed online at: https://drive.google.com/drive/folders/1EErNPuxByG0SeQPV6g6g_ LG-ghfzJj2Y?usp=sharing

\section{Conflicts of Interest}

The Authors declare no conflicts of interest regarding this study.

\section{Authors' Contributions}

GTT conceptualized the project and the methodology. EZ, with the support of AMK, analyzed the data, generated the tables and figures, and prepared the original draft under the supervision of FZ, MG and GTT. SCP and AKA performed the experimental procedures. All authors provided critical feedback, contributed to the manuscript, and approved the final version in accordance with criteria established by the International Committee of Medical Journal Editors (ICMJE).

\section{References}

1 Anderson WF, Jatoi I, Tse J and Rosenberg PS: Male breast cancer: a population-based comparison with female breast cancer. J Clin Oncol 28(2): 232-239, 2010. PMID: 19996029. DOI: $10.1200 / \mathrm{JCO} .2009 .23 .8162$

2 Siegel RL, Miller KD, Fuchs HE and Jemal A: Cancer statistics, 2021. CA Cancer J Clin 71(1): 7-33, 2021. PMID: 33433946. DOI: $10.3322 /$ caac. 21654

3 Speirs V and Shaaban AM: The rising incidence of male breast cancer. Breast Cancer Res Treat 115(2): 429-430, 2009. PMID: 18478326. DOI: 10.1007/s10549-008-0053-y

4 Abdelwahab Yousef AJ: Male breast cancer: Epidemiology and risk factors. Semin Oncol 44(4): 267-272, 2017. PMID: 29526255. DOI: 10.1053/j.seminoncol.2017.11.002

5 Liu N, Johnson KJ and Ma CX: Male breast cancer: an updated surveillance, epidemiology, and end results data analysis. Clin Breast Cancer 18(5): e997-e1002, 2018. PMID: 30007834. DOI: 10.1016/j.clbc.2018.06.013

6 Cardoso F, Bartlett JMS, Slaets L, van Deurzen CHM, van Leeuwen-Stok E, Porter P, Linderholm B, Hedenfalk I, Schröder C, Martens J, Bayani J, van Asperen C, Murray M, Hudis C, Middleton L, Vermeij J, Punie K, Fraser J, Nowaczyk M, Rubio IT, Aebi S, Kelly C, Ruddy KJ, Winer E, Nilsson C, Lago LD, Korde L, Benstead K, Bogler O, Goulioti T, Peric A, Litière S,
Aalders KC, Poncet C, Tryfonidis $\mathrm{K}$ and Giordano SH: Characterization of male breast cancer: results of the EORTC 10085/TBCRC/BIG/NABCG International Male Breast Cancer Program. Ann Oncol 29(2): 405-417, 2018. PMID: 29092024. DOI: $10.1093 /$ annonc/mdx651

7 Giordano SH, Cohen DS, Buzdar AU, Perkins G and Hortobagyi GN: Breast carcinoma in men: a population-based study. Cancer 101(1): 51-57, 2004. PMID: 15221988. DOI: 10.1002/cncr.20312

8 Vermeulen MA, Slaets L, Cardoso F, Giordano SH, Tryfonidis K, van Diest PJ, Dijkstra NH, Schröder CP, van Asperen CJ, Linderholm B, Benstead K, Foekens R, Martens JWM, Bartlett JMS and van Deurzen CHM: Pathological characterisation of male breast cancer: Results of the EORTC 10085/TBCRC/BIG/NABCG International Male Breast Cancer Program. Eur J Cancer 82: 219-227, 2017. PMID: 28292559. DOI: $10.1016 /$ j.ejca.2017.01.034

9 Piscuoglio S, Ng CK, Murray MP, Guerini-Rocco E, Martelotto LG, Geyer FC, Bidard FC, Berman S, Fusco N, Sakr RA, Eberle CA, De Mattos-Arruda L, Macedo GS, Akram M, Baslan T, Hicks JB, King TA, Brogi E, Norton L, Weigelt B, Hudis CA and Reis-Filho JS: The genomic landscape of male breast cancers. Clin Cancer Res 22(16): 4045-4056, 2016. PMID: 26960396. DOI: 10.1158/1078-0432.CCR-15-2840

10 Johansson I, Ringnér M and Hedenfalk I: The landscape of candidate driver genes differs between male and female breast cancer. PLoS One 8(10): e78299, 2013. PMID: 24194916. DOI: 10.1371/journal.pone.0078299

11 Johansson I, Nilsson C, Berglund P, Lauss M, Ringnér M, Olsson H, Luts L, Sim E, Thorstensson S, Fjällskog ML and Hedenfalk I: Gene expression profiling of primary male breast cancers reveals two unique subgroups and identifies $\mathrm{N}$ acetyltransferase-1 (NAT1) as a novel prognostic biomarker. Breast Cancer Res 14(1): R31, 2012. PMID: 22333393. DOI: $10.1186 /$ bcr 3116

12 Yeh E, Cunningham M, Arnold H, Chasse D, Monteith T, Ivaldi G, Hahn WC, Stukenberg PT, Shenolikar S, Uchida T, Counter CM, Nevins JR, Means AR and Sears R: A signalling pathway controlling $\mathrm{c}-\mathrm{Myc}$ degradation that impacts oncogenic transformation of human cells. Nat Cell Biol 6(4): 308-318, 2004. PMID: 15048125 . DOI: $10.1038 / \mathrm{ncb} 1110$

13 Zeidan BA, Townsend PA, Garbis SD, Copson E and Cutress RI: Clinical proteomics and breast cancer. Surgeon 13(5): 271-278, 2015. PMID: 25736469. DOI: 10.1016/j.surge.2014.12.003

14 Wang H, Shi T, Qian WJ, Liu T, Kagan J, Srivastava S, Smith RD, Rodland KD and Camp DG 2nd: The clinical impact of recent advances in LC-MS for cancer biomarker discovery and verification. Expert Rev Proteomics 13(1): 99-114, 2016. PMID: 26581546. DOI: 10.1586/14789450.2016.1122529

15 Senkus E, Kyriakides S, Ohno S, Penault-Llorca F, Poortmans P, Rutgers E, Zackrisson S, Cardoso F and ESMO Guidelines Committee: Primary breast cancer: ESMO Clinical Practice Guidelines for diagnosis, treatment and follow-up. Ann Oncol 26(Suppl 5): v8-30, 2015. PMID: 26314782. DOI: 10.1093/annonc/ mdv298

16 Anagnostopoulos AK, Stravopodis DJ and Tsangaris GT: Yield of 6,000 proteins by $1 \mathrm{D}$ nLC-MS/MS without pre-fractionation. J Chromatogr B Analyt Technol Biomed Life Sci 1047: 92-96, 2017. PMID: 27605470. DOI: 10.1016/j.jchromb.2016.08.031

17 Korovesi AG, Anagnostopoulos AK, Pierros V, Stravopodis DJ and Tsangaris GT: Normal mouse brain proteome II: Analysis of 
brain regions by high-resolution mass spectrometry. Cancer Genomics Proteomics 17(6): 757-767, 2020. PMID: 33099477. DOI: $10.21873 / \operatorname{cgp} .20230$

18 Mi H, Ebert D, Muruganujan A, Mills C, Albou LP, Mushayamaha $\mathrm{T}$ and Thomas PD: PANTHER version 16: a revised family classification, tree-based classification tool, enhancer regions and extensive API. Nucleic Acids Res 49(D1): D394-D403, 2021. PMID: 33290554. DOI: 10.1093/nar/gkaa1106

19 Tyanova S, Albrechtsen R, Kronqvist P, Cox J, Mann M and Geiger T: Proteomic maps of breast cancer subtypes. Nat Commun 7: 10259, 2016. PMID: 26725330. DOI: 10.1038/ncomms 10259

20 Yanovich G, Agmon H, Harel M, Sonnenblick A, Peretz T and Geiger T: Clinical proteomics of breast cancer reveals a novel layer of breast cancer classification. Cancer Res 78(20): 60016010, 2018. PMID: 30154156. DOI: 10.1158/0008-5472.CAN18-1079

21 Murphy CG, Mallam D, Stein S, Patil S, Howard J, Sklarin N, Hudis CA, Gemignani ML and Seidman AD: Current or recent pregnancy is associated with adverse pathologic features but not impaired survival in early breast cancer. Cancer 118(13): 32543259, 2012. PMID: 22086863. DOI: 10.1002/cncr.26654

22 Pozniak Y, Balint-Lahat N, Rudolph JD, Lindskog C, Katzir R, Avivi C, Pontén F, Ruppin E, Barshack I and Geiger T: Systemwide clinical proteomics of breast cancer reveals global remodeling of tissue homeostasis. Cell Syst 2(3): 172-184, 2016. PMID: 27135363. DOI: 10.1016/j.cels.2016.02.001

23 Suman S, Basak T, Gupta P, Mishra S, Kumar V, Sengupta S and Shukla Y: Quantitative proteomics revealed novel proteins associated with molecular subtypes of breast cancer. J Proteomics 148: 183-193, 2016. PMID: 27498393. DOI: 10.1016/j.jprot.2016.07.033

24 Calderón-González KG, Valero Rustarazo ML, Labra-Barrios ML, Bazán-Méndez CI, Tavera-Tapia A, Herrera-Aguirre ME, Sánchez del Pino MM, Gallegos-Pérez JL, González-Márquez H, Hernández-Hernández JM, León-Ávila G, Rodríguez-Cuevas S, Guisa-Hohenstein F and Luna-Arias JP: Determination of the protein expression profiles of breast cancer cell lines by quantitative proteomics using iTRAQ labelling and tandem mass spectrometry. J Proteomics 124: 50-78, 2015. PMID: 25918110. DOI: $10.1016 /$ j.jprot.2015.04.018

25 Liu F, Ma F, Wang Y, Hao L, Zeng H, Jia C, Wang Y, Liu P, Ong IM, Li B, Chen G, Jiang J, Gong S, Li L and Xu W: PKM2 methylation by CARM1 activates aerobic glycolysis to promote tumorigenesis. Nat Cell Biol 19(11): 1358-1370, 2017. PMID: 29058718. DOI: $10.1038 /$ ncb3630

26 Tveitarås MK, Selheim F, Sortland K, Reed RK and Stuhr L: Protein expression profiling of plasma and lungs at different stages of metastatic development in a human triple negative breast cancer xenograft model. PLoS One 14(5): e0215909, 2019. PMID: 31042781. DOI: 10.1371/journal.pone.0215909
27 Chahed K, Kabbage M, Hamrita B, Guillier CL, Trimeche M, Remadi S, Ehret-Sabatier L and Chouchane L: Detection of protein alterations in male breast cancer using two dimensional gel electrophoresis and mass spectrometry: the involvement of several pathways in tumorigenesis. Clin Chim Acta 388(1-2): 106-114, 2008. PMID: 17996735 . DOI: $10.1016 /$ j.cca.2007.10.018

$28 \mathrm{Key}$ statistics for breast cancer in men. Available at: https://www.cancer.org/cancer/breast-cancer-in-men/about/keystatistics.html [Last accessed on October 7, 2021]

29 Yu L, Zhao L, Wu H, Zhao H, Yu Z, He M, Jin F and Wei M: Moesin is an independent prognostic marker for ER-positive breast cancer. Oncol Lett 17(2): 1921-1933, 2019. PMID: 30675256. DOI: 10.3892/ol.2018.9799

30 Lamb R, Harrison H, Hulit J, Smith DL, Lisanti MP and Sotgia F: Mitochondria as new therapeutic targets for eradicating cancer stem cells: Quantitative proteomics and functional validation via MCT1/2 inhibition. Oncotarget 5(22): 11029-11037, 2014. PMID: 25415228. DOI: 10.18632/oncotarget.2789

31 Iino K, Mitobe Y, Ikeda K, Takayama KI, Suzuki T, Kawabata H, Suzuki Y, Horie-Inoue K and Inoue S: RNA-binding protein NONO promotes breast cancer proliferation by posttranscriptional regulation of SKP2 and E2F8. Cancer Sci 111(1): 148-159, 2020. PMID: 31733123. DOI: 10.1111/cas.14240

32 Talebizadeh Z, Simon SD and Butler MG: X chromosome gene expression in human tissues: male and female comparisons. Genomics 88(6): 675-681, 2006. PMID: 16949791. DOI: 10.1016/j.ygeno.2006.07.016

33 Tian HM, Liu XH, Han W, Zhao LL, Yuan B and Yuan CJ: Differential expression of filamin A and its clinical significance in breast cancer. Oncol Lett 6(3): 681-686, 2013. PMID: 24137390. DOI: 10.3892/ol.2013.1454

34 Caruso JA and Stemmer PM: Proteomic profiling of lipid rafts in a human breast cancer model of tumorigenic progression. Clin Exp Metastasis 28(6): 529-540, 2011. PMID: 21533873. DOI: 10.1007/s10585-011-9389-5

$35 \mathrm{Xu} \mathrm{Y,} \mathrm{Bismar} \mathrm{TA,} \mathrm{Su} \mathrm{J,} \mathrm{Xu} \mathrm{B,} \mathrm{Kristiansen} \mathrm{G,} \mathrm{Varga} \mathrm{Z,} \mathrm{Teng} \mathrm{L,}$ Ingber DE, Mammoto A, Kumar R and Alaoui-Jamali MA: Filamin A regulates focal adhesion disassembly and suppresses breast cancer cell migration and invasion. J Exp Med 207(11): 2421-2437, 2010. PMID: 20937704. DOI: 10.1084/jem.20100433

Received November 12, 2021

Revised December 29, 2021

Accepted December 31, 2021 